\section{\$20. Dynamo Efficiency and Beltrami Flows in Rectangular Cells}

Kageyama, A., Sato, T.

The magnetohydrodynamic (MHD) dynamo is the self-excitation of the magnetic field from electrically conducting fluid flows. Several groups are undertaking dynamo experiments using liquid metals aiming laboratory demonstration of the self-excitation of the magnetic field [1].The laboratory dynamo experiments have to be done under the constraint of small magnetic Reynolds number $R$ (order of 10) since the experimental apparatus size largely reflects on the costs (larger apparatus costs much more) and the maximum flow velocity (by a pump, for example) is limited. In order to make a successful dynamo experiment, it is important to design an "efficient" flow pattern under the condition of the limited low value of the magnetic Reynolds number $R$. Among a variety of flow patterns with the same flow kinetic energy, there must be a group of flows which is efficient for the dynamo action. In this work, we studied a kinematic dynamo problem in the low $R$ regime in a periodic box geometry. The growth rates for many different flows are compared with the same flow kinetic energy.

Some numerical calculations ([2],[3]) suggest that Beltrami flows are very efficient for the dynamo action. However, it is still unclear whether or not the Beltrami flow exactly gives the maximum peak efficiency of the dynamo since the number of compared flow patterns in the above papers are not sufficient. Much more elaborate survey is required for a decisive conclusion.

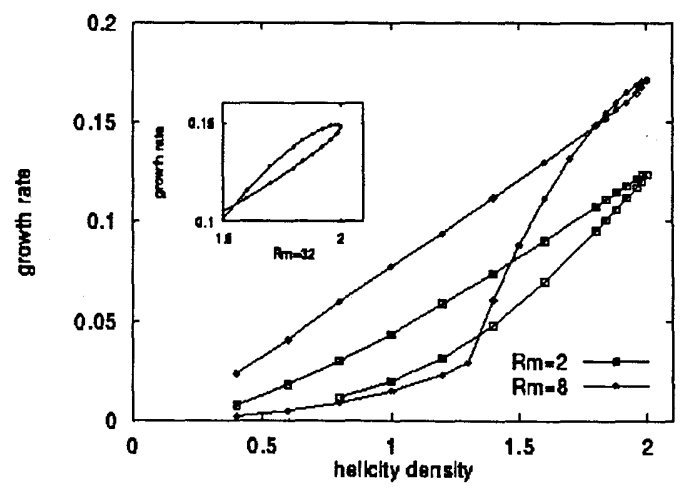

Figure 1: Magnetic field growth rate versus flow helicity in rectangular cell dynamo

We analyzed in detail the growth rate by the "Robertstype" flow defined by

$$
\begin{aligned}
& u_{x}=\frac{\sqrt{2}}{\sqrt{1+c^{2}}} \sin y \\
& u_{y}=\frac{\sqrt{2}}{\sqrt{1+c^{2}}} \sin x
\end{aligned}
$$

$$
u_{z}=\frac{c \sqrt{2}}{\sqrt{1+c^{2}}}(\cos x-\cos y) .
$$

This flow is a set of parallel helical flows and the pitch of the helical flow line is controlled by one parameter, $c$ in eqs. (1)-(3). Only when $c=1$, the velocity becomes a Beltrami flow. The flow helicity is the maximum in this case as a consequence of the general variational principle. Changing the pitch parameter $c$, we compared the dynamo growth rates and its correlation with the flow helicity. It is found that larger helicity flow always gives larger growth rate (Fig. 1). The most interesting finding by this analysis is that the maximum helicity flow exactly gives the maximum growth rate. This relation is shown by the sharp turns in the curves in Fig. $1(R=2$ and $R=8$ ).

This result indicates that the Beltrami flow is certainly the most efficient dynamo flow under the constraint of the constant kinetic energy, at least for low $R$ situation, which is our concern in the context of the laboratory dynamo experiment.

Care should be taken for the relation between the helicity and the Beltrami flow. In our model, $c=1$ (Beltrami) flow has the largest helicity. However, in general, one cannot get the maximum helicity flow under the constraint of the constant kinetic energy because the helicity may have infinitely large value by reducing the flow's spatial scale length. However, the magnetic field dissipation effect, which suppresses the dynamo growth rate, is enhanced in those cases. There must be an optimized helicity value for the dynamo efficiency. In addition, there are many qualitatively different Beltrami flow patterns which have exactly the same helicity. An example in a rectangular geometry is the Roberts flow [4] and the $A B C$ flow. These two flows are very different in their flow character but they may have exactly the same energy and helicity.

The details of this work is reported in [5].

\section{References}

1) A. Gailitis, Solar and Planetary Dynamos, M.R.E. Proctor, P.C. Matthews, and A.M. Rucklidge, ed., (Cambridge University Press, Cambridge) pp.91-98, 1993

2) T. Nakajima and M. Kono. Geophys. Astrophys. Fluid Dynamics, 60, 177 (1991)

3) F. Stefani, G. Gerbeth, and A. Gailitis, Velocity profile optimization for the Riga dynamo experiment, to appear in Fluid Mechanics and its applications, (Kluwer Academic Publishers, Dordrecht)

4) G.O. Roberts, Philos. Trans. R. Soc. London Ser.

A, 263, 93 (1972)

5) A. Kageyama and T. Sato Phys. of Plasmas, Vol.6, pp.771-776, 1999 